\title{
The use of $3^{\prime}$-deoxy- $3^{\prime}-18 \mathrm{~F}$-fluorothymidine (FLT) PET in the assessment of long-term survival in breast cancer patients treated with neoadjuvant chemotherapy
}

\author{
Benjamin E. Ueberroth ${ }^{1}$, Jawana M. Lawhorn-Crews ${ }^{1}$, Lance K. Heilbrun ${ }^{1,2}$, Daryn W. \\ Smith $^{2}$, Janice Akoury ${ }^{1}$, Rouba Ali-Fehmi ${ }^{1,2}$, Nicole T. Eiseler ${ }^{1,3}$, Anthony F. Shields ${ }^{1,2}$ \\ ${ }^{1}$ Wayne State University School of Medicine, 540 E Canfield St, Detroit, MI 48201, USA \\ ${ }^{2}$ Karmanos Cancer Institute, 4100 John R St, Detroit, MI 48201, USA \\ ${ }^{3}$ Cleveland Clinic Florida, 2950 Cleveland Clinic Blvd, Weston, FL 33331, USA
}

\section{Abstract}

Objective-To assess the role of serial FLT-PET scans during early neoadjuvant treatment as a prognostic marker of response to treatment and survival.

\begin{abstract}
Methods-This study is a prospective cohort study which draws from a larger original study which examined the utility of FLT-PET imaging across multiple cancers. Our cohort consisted of patients who had biopsy-confirmed breast cancer amenable to surgical resection. These patients underwent serial FLT-PET scans: the first scan prior to starting neoadjuvant chemotherapy (NAC), and a second scan shortly after starting NAC. SUV mean was derived using an isocontour ROI drawn approximately half way between the $\mathrm{SUV}_{\max }$ and background on three planes for each scan. The change in mean standardized uptake value $\left(\mathrm{SUV}_{\text {mean }}\right)$ for the primary tumor between these two scans was then calculated, and patients were stratified into "responder" and "nonresponder" groups based on a cut-off of $20 \%$ arithmetic decrease in $\mathrm{SUV}_{\text {mean }}$ between the two scans. The rates of pathologic complete response (pCR) on subsequent surgical excision, overall survival (OS), and progression-free survival (PFS) were then compared between the two groups to assess for significant difference between responders and non-responders.
\end{abstract}

Results-16 patients $(n=16)$ met criteria for inclusion and successfully underwent FLT-PET scans in the prescribed sequence of events. Seven of these patients had a decrease of $20 \%$ or larger between the two serial PET scans, making them "responders". The remaining nine patients were "non-responders" to NAC based on PET imaging. Between responders and non-responders, there was no significant difference in median PFS (7.9 years versus 3.7 years; $p=0.425)$ and median OS (7.5 years versus 5.0 years; $p=0.944)$. In the 14 patients who underwent surgical resection $(n=$ $14)$, there was no significant difference in the rate of achieving pCR (33\% vs. $14 \% ; p=0.5846)$ between responders and non-responders.

Conclusion-Further study of a larger sample size is needed to examine the potential role for FLT-PET in predicting response to neoadjuvant treatment, particularly in correlating with long-

Benjamin E. Ueberroth, fx4638@wayne.edu.

Publisher's Note Springer Nature remains neutral with regard to jurisdictional claims in published maps and institutional affiliations. 
term overall and progression-free survival. Our study is limited by small sample size, but does suggest that FLT-PET has a role in the long-term prognosis of breast cancer treated with NAC and surgical resection which is worthy of further study.

\section{Keywords}

Breast cancer; FLT-PET; Neoadjuvant; Survival

\section{Introduction}

Patients with large and locally advanced breast cancer commonly undergo treatment with neoadjuvant chemotherapy (NAC). The advantages of NAC include a higher probability of breast-conserving surgery and down-staging of disease prior to surgery [1]. Many of the benefits of NAC have been correlated with the confirmation of pathological complete response (pCR) upon post-NAC surgical resection of the breast tumor. In breast cancer, pCR itself has been strongly associated with longer survival and better outcomes overall, making it a desirable and useful endpoint in the setting of NAC [2,3]. By definition, pCR is determined once surgical resection is performed, and thus, other modalities must be evaluated in an effort to ascertain response to NAC prior to invasive surgery.

A variety of imaging modalities have been evaluated for determining the early response to NAC in breast cancer treatment, though it should be noted that there is no established standard for disease monitoring during active therapy [4]; ${ }^{18} \mathrm{~F}$-fluorodeoxyglucose positron emission tomography (FDG-PET) in particular has been well evaluated and documented for its role in prognosis of breast cancer patients treated with NAC [5-9]. On the other hand, PET studies examining novel tracers are still being refined. $3^{\prime}$-deoxy $-3^{\prime}-{ }^{18} \mathrm{~F}$ fluorothymidine (FLT) is a thymidine kinase substrate which has been well studied as a measure of proliferation and possible prognostic marker in other cancers [10-12] and, more importantly, has undergone studies for prognostic use in breast cancer [13-15]. While these studies support the use of FLT-PET in predicting pCR to NAC, few of these studies associate FLT-PET response early in NAC treatment to long-term outcomes, such as disease progression or survival. Pilot studies in the other malignancies have shown promise for early FLT-PET response as a prognostic tool for survival [16-18], however, few, if any, studies have been performed in breast cancer. FLT-PET is a known tool for predicting pCR in breast cancer and shows promise as an indicator of long-term outcomes in the other malignancies.

This study utilizes an independent cohort of female patients with breast cancer and data sets to validate the previous findings of Kostokaglu et al. [14] and with additional correlates. Our objective was to evaluate the role of serial FLT-PET scans in the setting of NAC as a prognostic indicator of best pathological response, progression-free survival (PFS), and overall survival (OS). We hypothesized that those patients with a $\mathrm{SUV}_{\text {mean }}$ decrease greater than $20 \%$ between a pretreatment scan (FLT1) and a scan after the first dose of NAC (FLT2) will have a better pathological response to NAC as well as longer PFS and OS. 


\section{Materials and methods}

\section{Patients and study design}

The data used in this study were collected under an investigator initiated pilot clinical study, which evaluated the utility of FLT-PET in response to therapy. The study was approved by the Institutional Review Board and the Radioactive Drug Research Committee at Wayne State University and started in 1999 (prior to the availability of ClinicalTrials.gov registration). All patients were recruited from the medical oncology clinics at Karmanos Cancer Institute, Detroit, Michigan. All patients signed written informed consent.

The present study employed in vitro measurements of tumor metabolism to predict and evaluate response to NAC. The primary objective was to associate the percent change in mean standardized uptake value $\left(\mathrm{SUV}_{\text {mean }}\right)$ between pre-therapy (FLT1) and after one cycle (FLT2) $\left(\%_{\triangle S U V m e a n}\right.$ FLT1-FLT2 $)$ of NAC with OS and PFS. SUV $\max$ was also considered and analyzed in the same context. A secondary objective was to associate change in SUV (mean and max) between FLT1 and FLT2 with pCR as a tool for monitoring NAC response prior to surgical resection. This was further evaluated in the context of "best pathological response", a combination of both complete and partial pathological response to NAC.

The eligibility criteria included histological confirmed breast cancer diagnosis, being a candidate for NAC and surgical resection of residual primary tumor after NAC at time of tissue diagnosis, and visible disease on $\mathrm{CT}$ and/or MR scans. The chemotherapy regimens chosen for each patient were not specified by study design and all patients included in this study were chemotherapy-naïve. Post-study treatment data were collected and are reported below (Table 1). Routine clinical treatment was provided by the treating oncologists postresection.

\section{Imaging and neoadjuvant chemotherapy protocol}

All patients who agreed to be placed on this protocol and who completed imaging according to the prescribed imaging scheme were included in analysis. The prescribed imaging scheme was as follows: patients were scheduled to undergo FLT1 between date of diagnosis and start date of NAC. Patients were then scheduled to undergo FLT2 after starting NAC.

From the pilot study patient roster, 21 patients met criteria by having breast cancer and were candidates for NAC and surgical resection. Of these patients, four never received a second FLT scan and, therefore, could not be evaluated in the context of this study. One patient received two PET scans, but did not receive NAC until after the second scan, and is, thus, not included in analysis. The remaining 16 patients underwent FLT1, NAC, and FLT2 in the correct sequencing and comprise the evaluable patient pool for this study.

No standardized length of time between FLT1 and starting NAC or starting NAC and FLT2 was outlined in the original study protocol. For the 16 patients included in analysis, the median time between FLT1 and FLT2 was 28 days. 14 of 16 patients had a period of 45 days or fewer between FLT1 and FLT2. Two patients had notably extended periods of time between FLT1 and FLT2 (69 days and 77 days) compared to the other patients; this was secondary to noncompliance with the scheduled follow-ups. These patients ultimately 
completed the imaging and NAC treatment in the correct sequence and are included in analysis.

\section{Tumor histology and immunohistochemistry}

The initial diagnosis of breast cancer was performed using a core needle biopsy sample prior to NAC. Estrogen receptor (ER), progesterone receptor (PR), and HER2/neu status were determined using immunohistochemical staining of samples obtained during post-NAC tumor resection. ER, PR, and HER2/neu status were each divided into negative, weakly positive, and strongly positive based on the field-standard levels of the proportion of cells displaying positive staining within each sample. Fluorescence in situ hybridization was utilized for one ambiguous case. All patients were included regardless of ER, PR, or HER2/neu status. Immunohistochemical profiles of the study population are available in Table 1.

\section{$3^{\prime}$-deoxy-3'-18 F-fluorothymidine positron emission tomography imaging}

Patients first underwent a pretreatment evaluation, including a CT or MR radiologic evaluation within 4 weeks of planned PET imaging showing measurable disease, physical examination, $\mathrm{CBC}$, and chemistry panel with electrolytes as part of standard care.

Patients were made comfortable in a supine position on the imaging table, and then injected via IV catheter with FLT standardized to body weight (mean 324 MBq; range 176-379 $\mathrm{MBq}$ ) with a specific activity of at least $3500 \mathrm{MBq} /$ microM. Vital signs were monitored at the beginning and end of scans. Periodic blood samples were taken during the imaging period for recording of blood activity measurements for later analysis. All patients were scanned on a calibrated Siemens EXACT/HR whole body tomography (Siemens Medical Solutions USA, Inc.) with a series of timed images starting with 60 min of dynamic imaging over the tumor. The dynamic image sequence consisted of 25 frames over $60 \mathrm{~min}(1 \times 60 \mathrm{~s}$, $4 \times 20 \mathrm{~s}, 4 \times 40 \mathrm{~s}, 4 \times 60 \mathrm{~s}, 4 \times 180 \mathrm{~s}$, and $8 \times 300 \mathrm{~s}$ ) followed by static imaging of the rest of the body for up to $120 \mathrm{~min}$.

Image interpretation was based on semi-quantitative analysis using computer programs developed in IDL (ITT Visual Information Solutions) along with the clinical application programing package (CTI/Siemens). A visual inspection of the images was preformed to detect uptake in tumors and normal tissues, and confirmed with either CT or MRI followed by quantitation by the method of standardized uptake value in a region of interest (ROI). SUV is defined as the activity per gram in the tissue divided by the activity per gram in the whole body.

ROIs were drawn over the primary breast tumor in the plane with the highest SUV and the two adjacent planes (Fig. 1). The $\mathrm{SUV}_{\max }$ was defined as the average of the most active pixel for the three planes. $\mathrm{SUV}_{\text {mean }}$ was drawn using an isocontour drawn approximately half way between the $\mathrm{SUV}_{\max }$ and background on the three planes for each scan. The percentage change in $\mathrm{SUV}_{\text {mean }}$ and $\mathrm{SUV}_{\max }$ was calculated for the scans of each patient: $\% \Delta \mathrm{SUV}_{\text {mean_FLT1 vs FLT2 }}$ and $\% \Delta \mathrm{SUV}_{\text {max_FLT1 vs FLT2 }}$; respectively [19]. 


\section{Treatment}

All patients received NAC on the basis of an expected subsequent surgical treatment, lumpectomy versus modified radical mastectomy. The NAC regimens were standard regimens chosen by the treating physician and are described in Table 1. After completion of NAC, 14 of 16 patients underwent surgical treatment. Two patients progressed rapidly and ultimately expired after starting NAC but before undergoing surgical treatment. All 14 surgically treated patients had concurrent axillary lymph-node dissection. Due to the immunohistochemical heterogeneity, patients underwent various combinations of adjuvant chemotherapy, radiation therapy, and/or hormonal therapy (Table 1).

\section{Pathology assessment and patient outcome}

Pathological complete response (pCR) was defined as no evidence of residual invasive cancer in the resected breast tissue specimen and lymph nodes. Partial pathological response $(\mathrm{pPR})$ was defined as decreased tumor size at time of surgical resection versus size demonstrated on staging CT scan prior to NAC. Pathological response data were taken directly from surgical pathology reports within the patient's electronic medical record (EMR). Patients were followed in the EMR, public records, and by contact when necessary to determine if they were alive and still in remission.

\section{Statistical analysis}

To analyze the association of OS or PFS with change in SUV mean between FLT1 and FLT2, patients were split into two groups termed "responders" and "non-responders" based on a

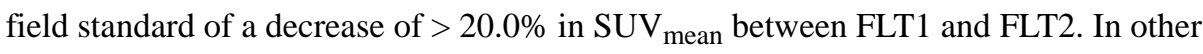

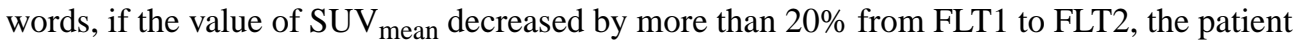
was considered a "responder" to NAC based on FLT-PET. All other patients were termed "non-responders", including those with an increase in the value of SUV $\mathrm{Sean}_{\text {from FLT1 to }}$ FLT2 and those with a decrease in SUV mean from FLT1 to FLT2 between 0\% and 20.0\%. Nine patients were classified as responders, and the remaining seven patients as nonresponders. Of note, the nine patients classified as responders using $\% \Delta \mathrm{SUV}_{\text {mean }}$ were found to be the same when using $\% \Delta \mathrm{SUV}_{\max }$ at the same cut-off of $-20.0 \%$. A separate analysis using $\mathrm{SUV}_{\max }$ is, thus, not included due to that redundancy.

Since the change in SUV could not be known until after FLT2, the landmark method was used to delineate the start of the follow-up period. The longest period between FLT1 and FLT2 was 77 days. Thus, a landmark time of 80 days from FLT1 was used to standardize the start of the follow-up period for all patients at a time when change in SUV was known. OS and PFS were calculated from each patient's respective FLT1 date and the landmark time of 80 days was then subtracted from the final OS and PFS times. There was no loss of patients due to the landmark analysis.

Standard Kaplan-Meier analyses of PFS and OS were performed separately as described above using responders and non-responders. Kaplan-Meier analysis of OS and PFS was also performed for all 16 patients as a single population. The relationships between PET response and PFS as well as PET response and OS were analyzed using log-rank testing between the responder and non-responder groups. 
Cox proportional hazard regression model landmark analyses were performed to estimate the degree of event risk for PET responders versus non-responders. First, discrete analyses based on PET response status were performed. Hazard ratios with $80 \%$ confidence intervals

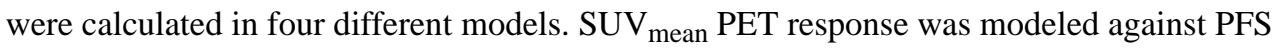
and OS in two separate analyses. SUV $\mathrm{Sax}_{\max }$ PET response was also modeled against PFS and OS in two separate analyses.

Furthermore, the Cox regression model was also used to analyze PFS and OS as a function of continuous ungrouped percent change in SUV. The Cox model was, thus, fit to the entire group of 16 patients as a single-study population.

Finally, complete pathological response (pCR) and best pathological response (BPR) were evaluated for a relationship to percent change between $\mathrm{SUV}_{\text {mean }}$ of FLT1 and FLT2. Pathological complete response is exclusively defined by the absence of tumor cells on the surgical specimen during post-NAC surgical excision. BPR is based on clinicopathologic criteria, typically also using the pathology report for the surgically removed specimen postNAC. However, as noted below, in the face of obvious clinical evidence of disease progression, some patients were deemed to have progressive disease without pathology specimen. The possible results for pathological response are described as complete response, partial response, stable disease, or progressive disease. Best pathological response is the combined outcome of either complete or partial pathological response based on surgical specimen pathology.

Wilson-type, two-sided $80 \%$ confidence intervals (CIs) were computed for rates, which were compared by Fisher's exact test. Odds ratios were generated for patients dichotomized into groups of greater than or less than $-20.0 \%$ change in SUV mean between FLT1 and FLT2. In this manner, serial FLT-PET scans could be evaluated for possible value in predicting pathological response to NAC based on a cut-off of $-20.0 \%$ change in $\mathrm{SUV}_{\text {mean }}$ between FLT1 and FLT2.

It is important to note that, due to the small sample size of this study, CIs at the $80 \%$ confidence level are provided and all the results must be considered accordingly. Analyses were performed using SAS 9.4 and Stata 15 software.

\section{Results}

From November 1999 to December 2002, 21 patients with breast cancer confirmed by biopsy underwent FLT-PET scanning before starting NAC. Four patients were not evaluable in our analysis due to not receiving a second FLT-PET scan, so no change in SUV parameters could be calculated. All 16 patients with multiple scans are included in analysis. Table 2 provides an outline of the results, including confidence levels and intervals.

Patients were identified as candidates for NAC and subsequent surgical treatment at the time of tissue diagnosis. Fourteen patients ultimately underwent surgical treatment; the two other patients expired prior to undergoing surgery. Based on the post-NAC surgical resection specimens, four of fourteen surgically treated patients reached pCR. Seven patients had a pPR. Three patients had stable disease. Two patients did not have a documented post-NAC 
biopsy to assess pathological response; however, both of these patients suffered disease progression on clinical grounds and were, thus, included in the "progressive disease" group.

Considering all patients in the study population $(n=16)$, the median PFS was 5.7 years and median OS was 6.3 years.

\section{Association of FLT-PET response status and PFS}

As above, "response" was defined as a $\mathrm{SUV}_{\text {mean }}$ change arithmetically less than $-20.0 \%$ between FLT1 and FLT2. For responders, the median PFS was 7.9 years versus 3.7 years in non-responders. The 1-year PFS rate was $70 \%$ for responders and $68 \%$ for non-responders (Table 3). The 5-year PFS rate was 58\% for responders and 33\% for non-responders (Fig. 2) (Table 3).

Duration of PFS did not differ significantly by FLT-PET response status $(p=0.425)$.

\section{Association of FLT-PET response status and OS}

Responders demonstrated a median OS of 7.5 years versus 5.0 years for non-responders. The 1 -year OS rate for responders was $88 \%$ versus $70 \%$ in non-responders (Table 3). The 5-year OS rate for responders was $59 \%$ versus $51 \%$ in non-responders (Fig. 3).

Duration of OS did not differ significantly by FLT-PET response status $(p=0.944)$.

\section{Cox regression analysis of PFS and OS by PET response status}

The first Cox regression model examined $\mathrm{SUV}_{\text {mean }}$ PET response grouping versus PFS. The progression hazard ratio $(\mathrm{HR})=0.605$ with an $80 \%$ CI of $(0.267,1.368)$.

The second Cox regression model examined SUV $\mathrm{max}_{\max }$ PET response and PFS. Since the dichotomy of responders and non-responders is identical when using $\mathrm{SUV}_{\max }$, these results are identical, with an $\mathrm{HR}=0.605$ and the same $80 \%$ CI.

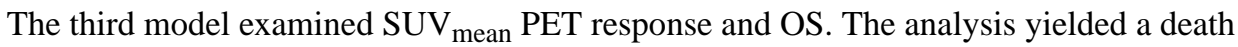
hazard ratio $(\mathrm{HR})=0.955$ with an $80 \% \mathrm{CI}$ of $(0.417,2.191)$.

The fourth and final Cox regression model examined $\mathrm{SUV}_{\max }$ PET response and OS. As with PFS, the OS results for $\mathrm{SUV}_{\text {mean }}$ and $\mathrm{SUV}_{\max }$ dichotomies are identical.

\section{Cox regression analysis of continuous ungrouped \%change in $\mathrm{SUV}_{\text {mean }}$}

The Cox model was fit to the entire group of 16 patients as a whole. When examining $\mathrm{SUV}_{\text {mean }}$, this yielded a progression hazard ratio $(\mathrm{HR})=0.994$ (with $80 \% \mathrm{CI}=0.979-1.010$ ) for each one unit (i.e., percentage point) increase in the \% change from FLT1 to FLT2.

A similar Cox model analysis yielded a death hazard ratio $(\mathrm{HR})=0.992$ (with $80 \% \mathrm{CI}=$ $0.977-1.007$ ) for each one unit (i.e., percentage point) increase in the $\%$ change from FLT1 to FLT2. 


\section{Association of PET response by $\mathrm{SUV}_{\text {mean }}$ to $\mathrm{PCR}$}

Nine patients had an SUV mean decrease larger than $20 \%$ between FLT1 and FLT2, termed "responders" as above (Table 4). Seven patients had an $\operatorname{SUV}_{\text {mean }}$ decrease $<20 \%$ between the two scans ("non-responders"). Three out of nine PET responders (33\%) demonstrated pCR yielding an $80 \% \mathrm{CI}=0.17-0.55$. One out of seven non-responders $(14 \%)$ had $\mathrm{pCR}$, yielding an $80 \% \mathrm{CI}=0.04-0.38$. These $\mathrm{pCR}$ rates were not statistically significantly different ( $p=0.5846$ by Fisher's exact test). Logistic regression modeling yielded an odds ratio of 3.00 , but with a large $80 \% \mathrm{CI}=0.57-15.7$.

\section{Association of PET response by $\mathrm{SUV}_{\text {mean }}$ to best pathologic response}

Best pathologic response (BPR) is defined in this analysis as the combined outcomes of pCR and pPR (Table 4). Eight out of nine "responders" (89\%) demonstrated at least pPR, yielding an $80 \% \mathrm{CI}=0.69-0.97$. Three out of seven "non-responders" $(43 \%)$ reached a BPR, yielding an $80 \% \mathrm{CI}=0.23-0.66$. These BPR rates were not statistically significantly different ( $p=0.1056$ by Fisher's exact test). Logistic regression modeling yielded an odds ratio of 10.67 , but with a large $80 \% \mathrm{CI}=2.00-56.1$.

\section{Discussion}

We present a prospective study examining the utility of pretreatment and early treatment serial FLT-PET scans in assessing OS, PFS, and pCR in the setting of NAC for the treatment of breast cancer. While our statistical significance was severely limited by small sample size, our study does provide a cohort of breast cancer patients followed for over a decade for OS and PFS. This provides a long-term outlook on breast cancer survival for those treated with NAC and surgery, which, in most studies, does not extend beyond 1- or 5-year survival rates. PFS (7.9 years versus 3.7 years) and OS (7.5 years versus 5.0 years) were both numerically longer in FLT-PET responders versus non-responders, but not significantly so. Responders did demonstrate higher rates of BPR compared to non-responders (OR 10.67; $p=0.1056$ ), which approached a $90 \%$ significance level; while this is not significant at the desired $95 \%$ significance level, it is worth noting that there was a difference here even with the extremely small sample size; further study is needed to better examine FLTPET in predicting pathologic response to NAC, which has been suggested but not confirmed here. Responders also demonstrated more than double the likelihood relative to non-responders for achieving pCR to NAC; however, this was not significant. The above results held the same for using both $\mathrm{SUV}_{\text {mean }}$ and $\mathrm{SUV}_{\max }$ to classify and evaluate patients. Further study on a larger sample of patients is necessary to better validate these results, with our less stringent confidence level providing suggestion that FLT-PET may have a role in this setting.

With this in mind, molecular imaging has been proven effective in assessing both early response to chemotherapy as well as long-term outcomes in breast cancer patients treated with neoadjuvant chemotherapy. Molecular imaging has not, however, been adopted as a standard for monitoring disease during active therapy. Multiple groups have demonstrated the utility of FDG-PET imaging in evaluating the early response of patients with breast cancer of mixed phenotypes to neoadjuvant chemotherapy and associating this response with long-term outcomes [5-8]. Molecular tracers alternative to FDG are being investigated for 
similar uses in the setting of neoadjuvant chemotherapy. FLT-PET imaging has shown promise in solid organ tumors for evaluating response to treatment in the form of pathological complete response, as well as associating with long-term outcomes such as overall survival [16, 17]. Furthermore, in breast cancer, FLT-PET has been shown to be useful in predicting pathologic complete response to neoadjuvant chemotherapy [13, 14]. However, there is a lack of studies examining the use of FLT-PET in evaluating long-term outcomes for breast cancer patients treated with neoadjuvant chemotherapy.

We present a study with long-term follow-up on the order of years for breast cancer patients treated with NAC and surgical resection, a relatively lengthy period of time for follow-up in a study related to malignancy. Furthermore, while FDG-PET is well vetted for evaluating treatment response in breast cancer and FLT-PET has been examined similarly in the other cancers, we evaluate FLT-PET in the setting of breast cancer treated with NAC, for which there are no other direct studies. This combination of long-term follow-up and possible novel application of FLT-PET provides the value of our study.

As noted above, this study does suffer from some limitations, most notably small sample size; the small sample size limits the overall interpretation of the results in exploring FLTPET as a predictor of survival in these patients. Furthermore, two patients who otherwise met criteria for this study expired prior to undergoing a second FLT-PET scan, making them unevaluable for this study, which depends on changes between PET scans; this could potentially have led to attrition bias, however, again, given the lack of significant results and small sample size to begin with, this is mentioned here for posterity. In terms of the sequence of events, there was no specific time frame or number of chemotherapy doses between the start of NAC (e.g. first dose) and undergoing the second FLT-PET scan; some patients underwent this as shortly as a few days after starting NAC, while two patients did not undergo their second PET scan until 9-10 weeks after starting NAC. These disparities in NAC-to-FLT2 intervals could impart some effect on the uptake of the second PET scan, in turn, affecting the results. Finally, while the original FLT-PET study from which these data are drawn was prospective, the therapy chosen was left to the treating physicians. This led to a mixture of applied therapies. While this may make our results more generalizable, it limits the ability to evaluate any particular treatment.

With these limitations in mind, there is at least some suggestion, between the current literature and our small study, that FLT-PET can be utilized during NAC treatment for breast cancer to assess the early response as it relates to long-term outcomes, including pathological response and survival. Further study with a larger sample size may elucidate FLT-PET as a viable method for stopping ineffective NAC treatment, limiting unnecessary chemotherapy toxicity, and allowing more rapid transition to a possibly effective second-line treatment. Further research is warranted on a larger sample size.

\section{Conclusions}

Breast cancer patients treated with NAC demonstrate measurable long-term progression-free and overall survival and this is the standard of care for many patients with locally advanced disease. Our results suggest a possible use of pretreatment and early NAC treatment FLT- 
PET scans in predicting both progression-free and overall survival in breast cancer patients, as well as a pathological response on subsequent surgical excision. Given the lack of significance and limitations of very small sample size, further study is warranted on a larger scale to evaluate the use of FLT-PET in guiding NAC treatment decisions in the setting of breast cancer.

\section{Acknowledgements}

No potential conflicts of interest were disclosed.

\section{References}

1. Untch M, Konecny GE, Paepker S, von Minckwitz. Current and future role of neoadjuvant therapy for breast cancer. Breast. 2014;23(5):526-37. [PubMed: 25034931]

2. Cortazar P, Zhang L, Untch M, Mehta K, Costantino JP, Wolmark N, et al. Pathological complete response and long-term clinical benefit in breast cancer: the CTNeoBC pooled analysis. Lancet. 2014;384(9938):164-72. [PubMed: 24529560]

3. Cortazar P, Geyer CE. Pathological complete response in neoadjuvant treatment of breast cancer. Ann Surg Oncol. 2015;22(5):1441-6. [PubMed: 25727556]

4. Teshome M, Kuerer HM. Neoadjuvant therapy in the treatment of breast cancer. Surg Oncol Clin N Am. 2014;23(3):505-23. [PubMed: 24882348]

5. Carkaci S, Sherman CT, Ozkan E, Adrada BE, Wei W, Rohren EM, et al. ${ }^{(18)}$ F-FDG PET/CT predicts survival in patients with inflammatory breast cancer undergoing neoadjuvant chemotherapy. Eur J Nucl Med Mol Imaging. 2013;40(12):1809-16. [PubMed: 23877633]

6. Champion L, Lerebours F, Alberini JL, Fourme E, Gontier E, Bertrand F, et al. ${ }^{18}$ F-FDG PET/CT to predict response to neoadjuvant chemotherapy and prognosis in inflammatory breast cancer. J Nucl Med. 2015;56(9):1315-21. [PubMed: 26159587]

7. Kolsenikov-Gauthier H, Vanlemmens L, Baranzelli MC, Vennin P, Servent V, Fournier C, et al. Predictive value of neoadjuvant chemotherapy failure in breast cancer using FDG-PET after the first course. Breast Cancer Res Treat. 2012;131(2):517-25. [PubMed: 22037787]

8. Lim I, Noh WC, Park J, Park JA, Kim HA, Kim EK, et al. The combination of FDG PET and dynamic contrast-enhanced MRI improves the prediction of disease-free survival in patients with advanced breast cancer after the first cycle of neoadjuvant chemotherapy. Eur J Nucl Med Mol Imaging. 2014;41(10):1852-60. [PubMed: 24927797]

9. Groheux D, Martineau A, Teixeira L, Espie M, de Cremoux P, Bertheau P, et al. ${ }^{(18)}$ FDG-PET/CT for predicting the outcome in ER+/HER2- breast cancer patients: comparison of clinicopathological parameters and PET image-derived indices including tumor texture analysis. Breast Cancer Res. 2017;19(1):3. [PubMed: 28057031]

10. McKinley ET, Watchmaker JM, Chakravarthy AB, Meyerhardt JA, Engelman JA, Walker RC, et al. [(18)F]-FLT PET to predict early response to neoadjuvant therapy in KRAS wild-type rectal cancer: a pilot study. Ann Nucl Med. 2015;29(6):535-42. [PubMed: 25899481]

11. Rendl G, Rettenbacher L, Holzmannhofer J, Datz L, Jauser-Kronberger C, Fastner G, et al. Assessment of response to neoadjuvant radiochemotherapy with F-18 FLT and F-18 FDG PET/CT in patients with rectal cancer. Ann Nucl Med. 2015;29(3):284-94. [PubMed: 25503812]

12. Benz MR, Czernin J, Allen-Auerbach MS, Dry SM, Sutthiruangwong P, Spick C, et al. 3' deoxy $-3^{\prime}-[18 \mathrm{~F}]$ fluorothymidine positron emission tomography for response assessment in soft tissue sarcoma: a pilot study to correlate imaging findings with tissue thymidine kinase 1 and Ki-67 activity and histopathologic response. Cancer. 2012;118(12):3135-44. [PubMed: 22020872]

13. Crippa F, Agresti R, Sandri M, Mariani G, Padovano B, Alessi A, et al. ${ }^{18}$ F-FLT PET/CT as an imaging tool for early prediction of pathological response in patients with locally advanced breast cancer treated with neoadjuvant chemotherapy: a pilot study. Eur J Nucl Med Mol Imaging. 2015;42(6):818-30. [PubMed: 25673053] 
14. Kostakoglu L, Duan F, Idowu MO, Jolles PR, Bear HD, Muzi M, et al. A phase II study of 3' deoxy-3'-18F-fluorothymidine PET in the assessment of early response of breast cancer to neoadjuvant chemotherapy: results from ACRIN 6688. J Nucl Med. 2015;56(11):1681-1689. [PubMed: 26359256]

15. Woolf DK, Beresford M, Li SP, Dowsett M, Sanghera B, Wong WL, et al. Evaluation of FLT-PET$\mathrm{CT}$ as an imaging biomarker of proliferation in primary breast cancer. Br J Cancer. 2014;110(12): 2847-2854 [PubMed: 24832174]

16. Chao KS Functional imaging for early prediction of response to chemoradiotherapy: $3^{\prime}$-deoxy-3' -18 F-fluorothymidine positron emission tomography—a clinical application model of esophageal cancer. Semin Oncol.2006; 33(6 Suppl 11):59-63.

17. Dehdashti F, Grigsby PW, Myerson RJ, Nalbantoglu I, Ma C, Siegel BA Positron emission tomography with [(18)F]-3' -deoxy-3' fluorothymidine (FLT) as a predictor of outcome in patients with locally advanced resectable rectal cancer: a pilot study. Mol Imaging Biol. 2013;15(1):106113. [PubMed: 22684813]

18. Ott K, Hermann K, Schuster T, Langer R, Becker K, Wieder HA, et al. Molecular imaging of proliferation and glucose utilization: utility for monitoring response and prognosis after neoadjuvant therapy in locally advanced gastric cancer. Ann Surg Oncol. 2011;18(12):3316-3323. [PubMed: 21537865]

19. Shields AF, Briston DA, Chandupatla S, Douglas KA, Lawhorn-Crews J, Collins JM, et al. A simplified analysis of $[18 \mathrm{~F}] 3^{\prime}$-deoxy-3' ${ }^{\prime}$-fluorothymidine metabolism and retention. Eur J Nucl Med Mol Imaging. 2005;32(11):1269-1275. [PubMed: 15991018] 
a FLT Responder

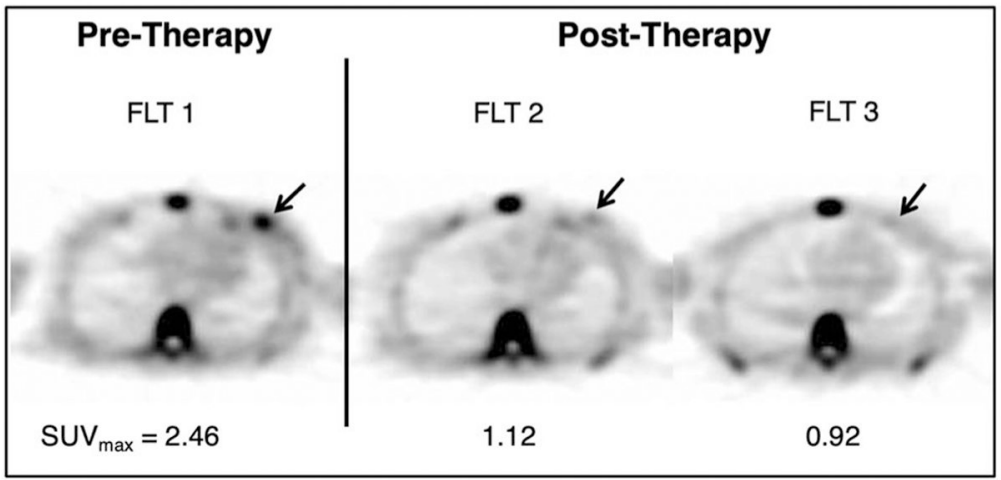

b FLT Non-responder

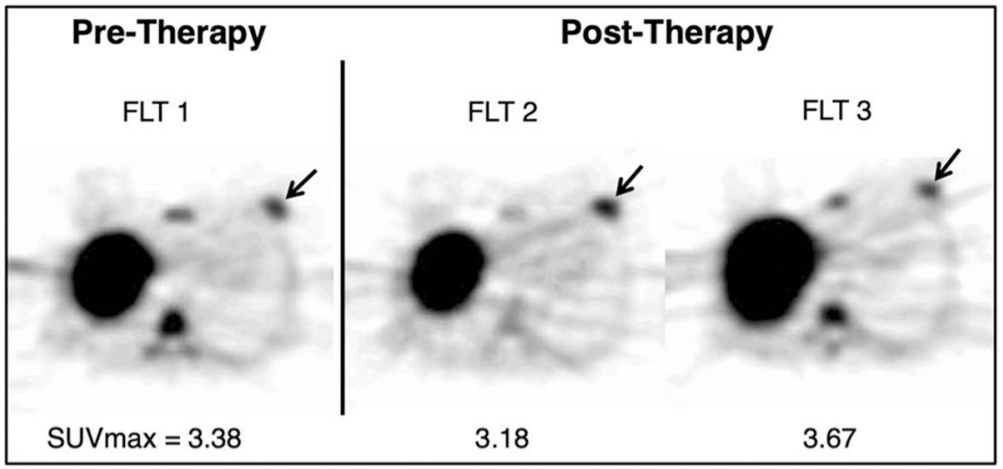

Fig. 1.

${ }^{18}$ F-FLT positron emission tomography axial images depict maximum standard uptake values in pre- and post-therapy patients. The tumor site represents a pathological response over three ${ }^{18}$ F-FLT-PET scans. Physiologic FLT uptake is also visible in the sternal marrow, spine, and liver 

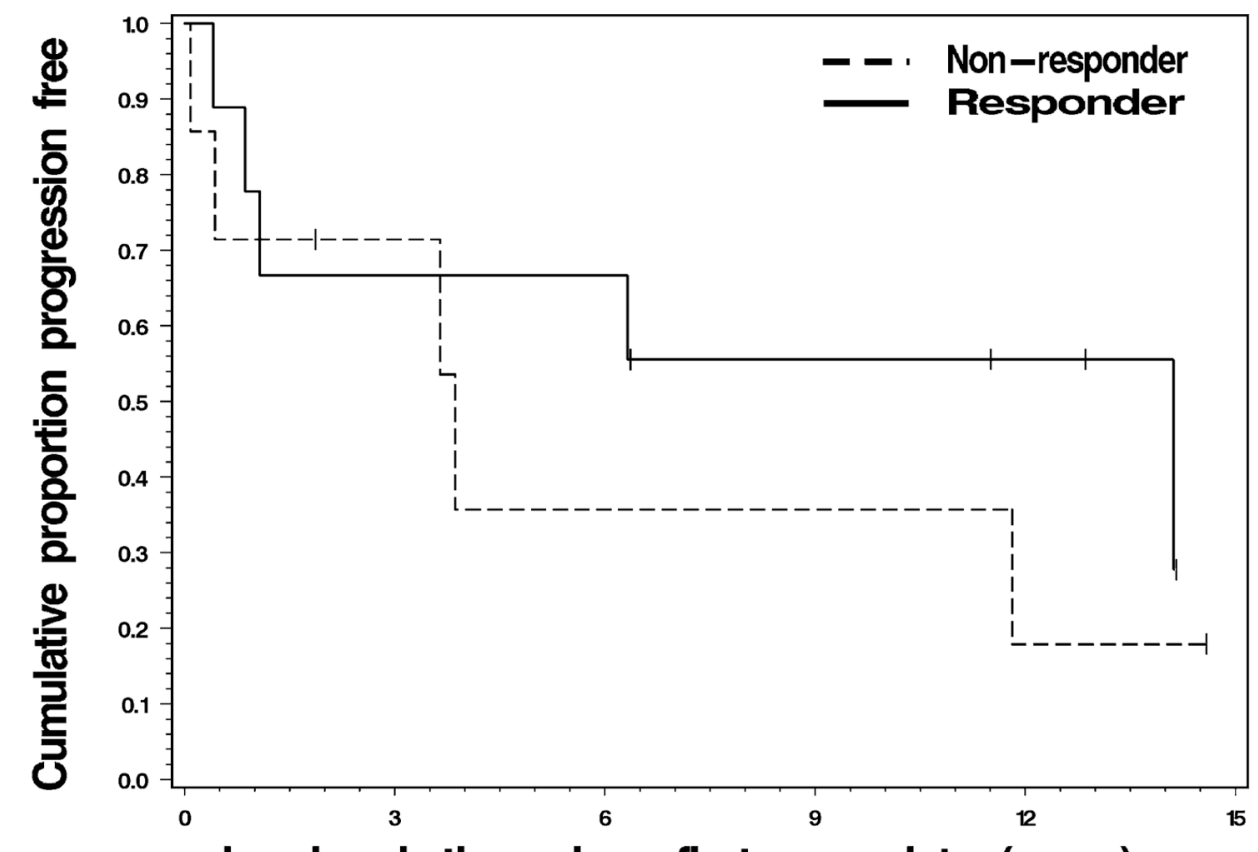

Patients Landmark time since first scan date (years)
at risk

Non-resp. 7

4
6

2

1
3

Fig. 2.

Progression free survival for responders versus non-responders based on pre- and early treatment FLT-PET scans 


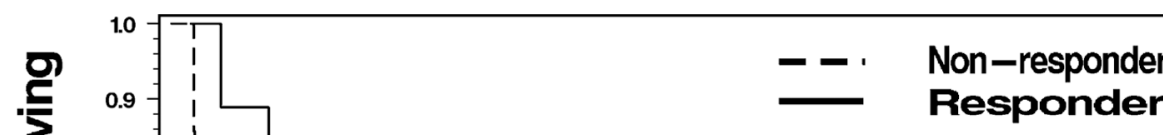

\section{at risk}

Non-resp.

Responder

.

!
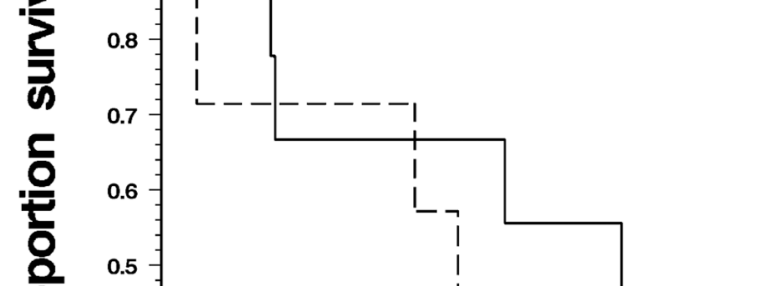

응 0.

\ $0.3=$

일 0.2

흥

0.1

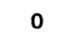

10

Fig. 3.

Overall survival for responders versus non-responders based on pre- and early treatment FLT-PET scans 


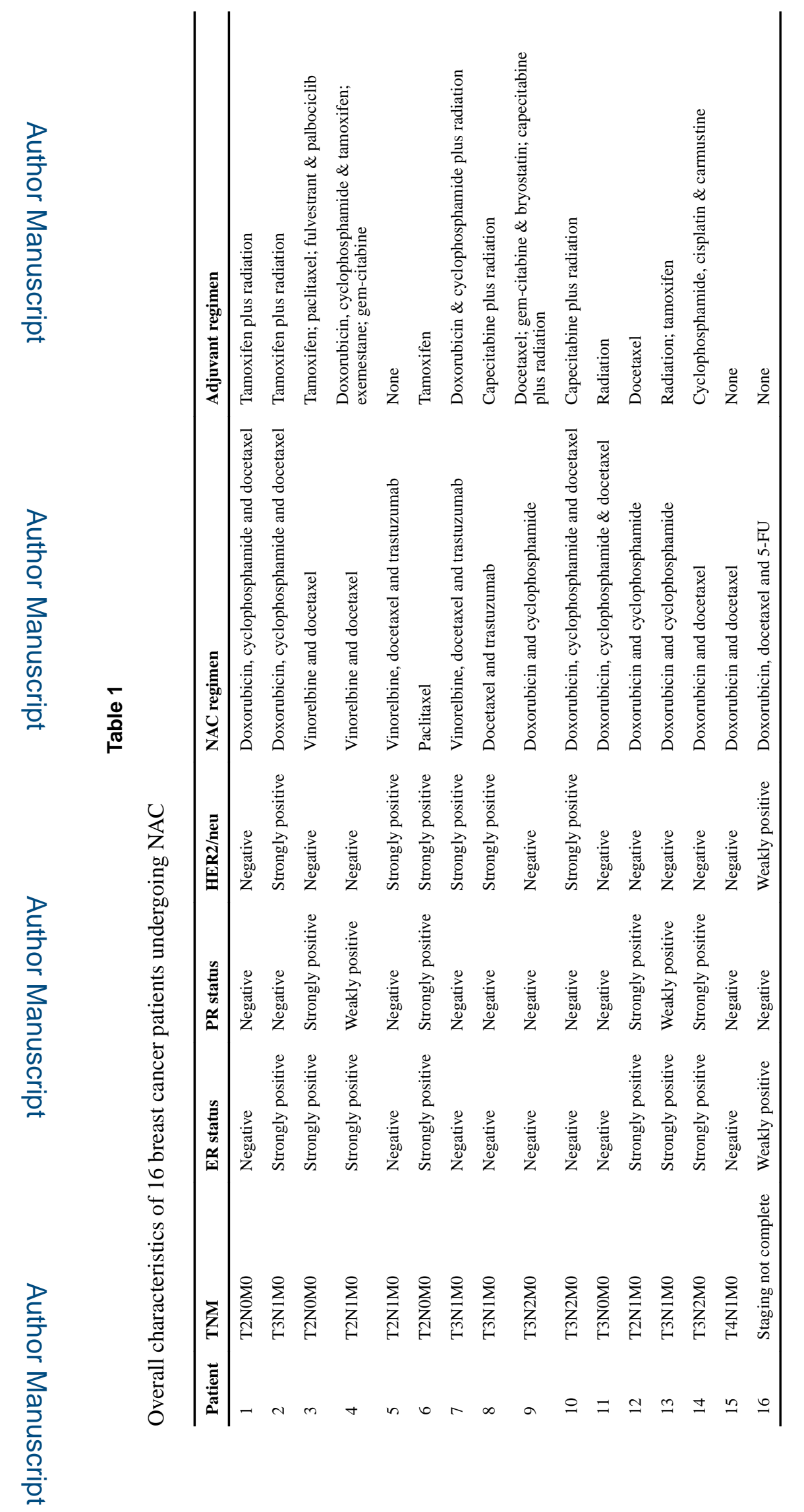

Ann Nucl Med. Author manuscript; available in PMC 2020 June 01. 


\section{롤 \\ 일}

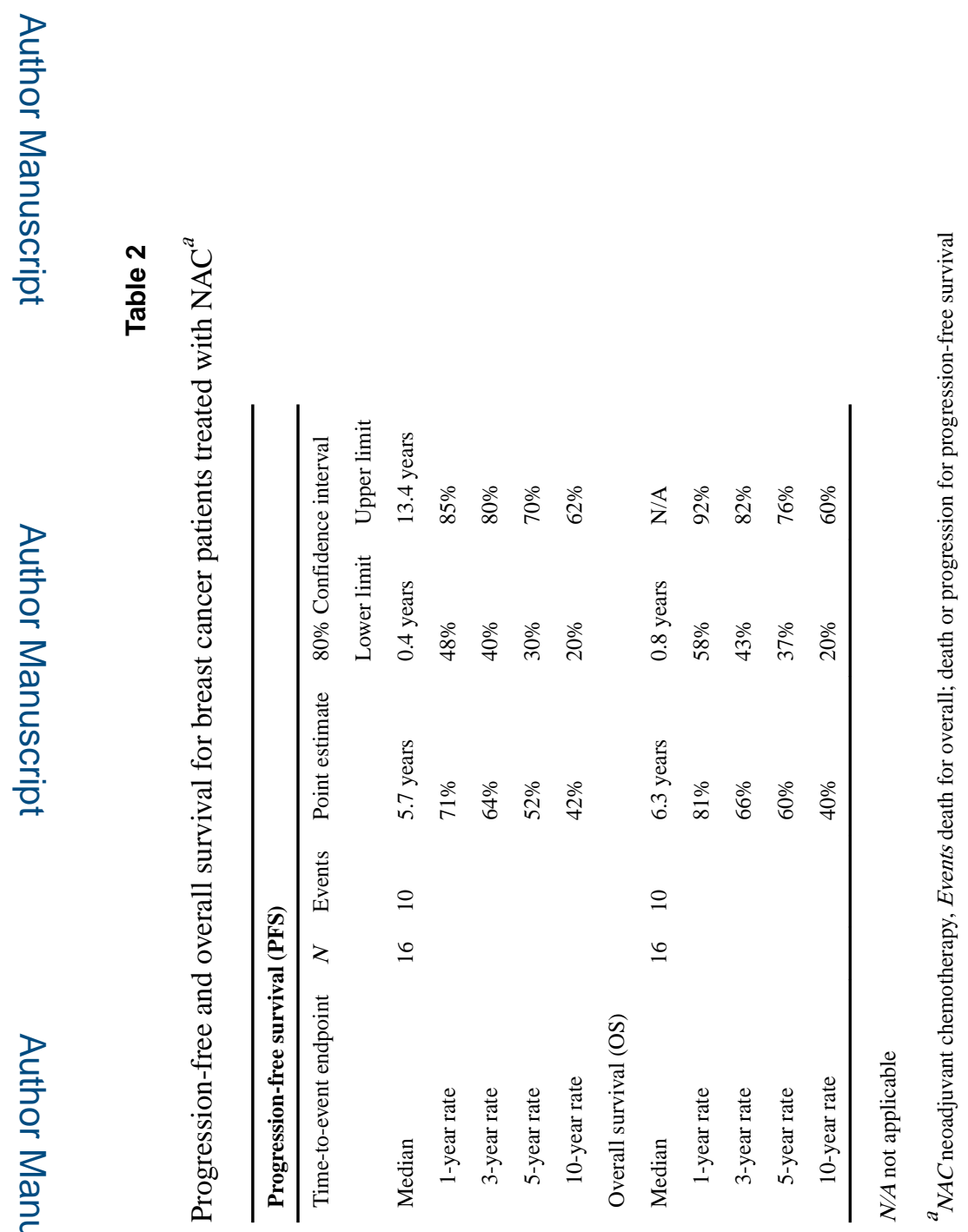




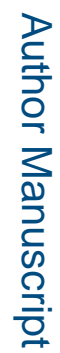

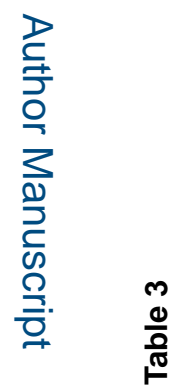

룰

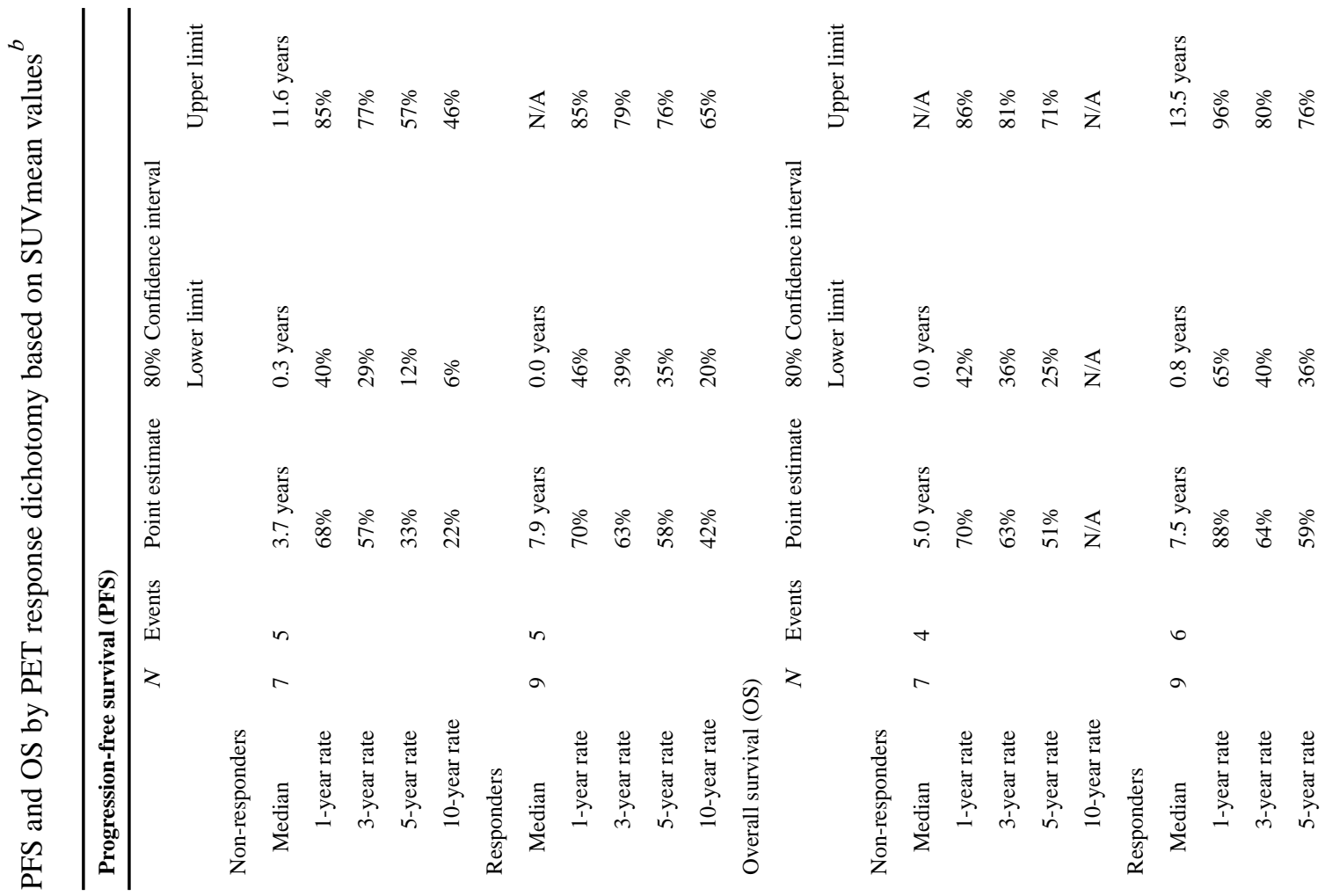




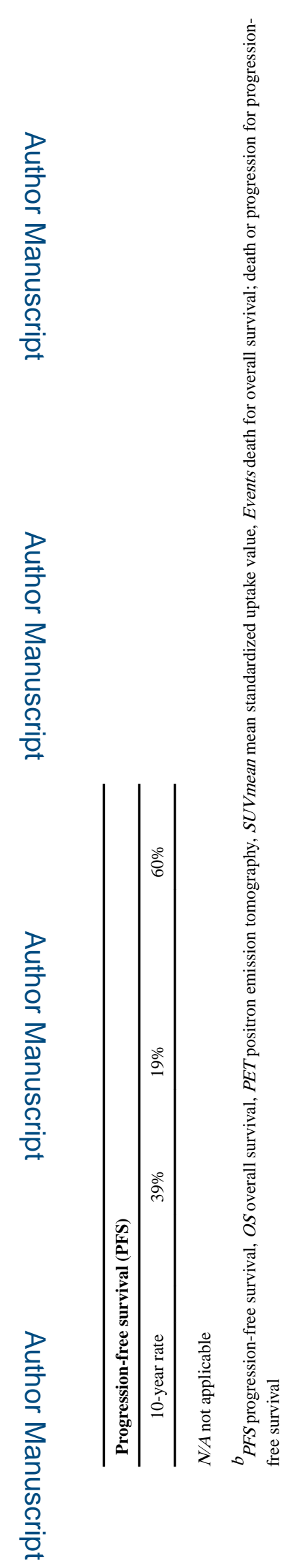



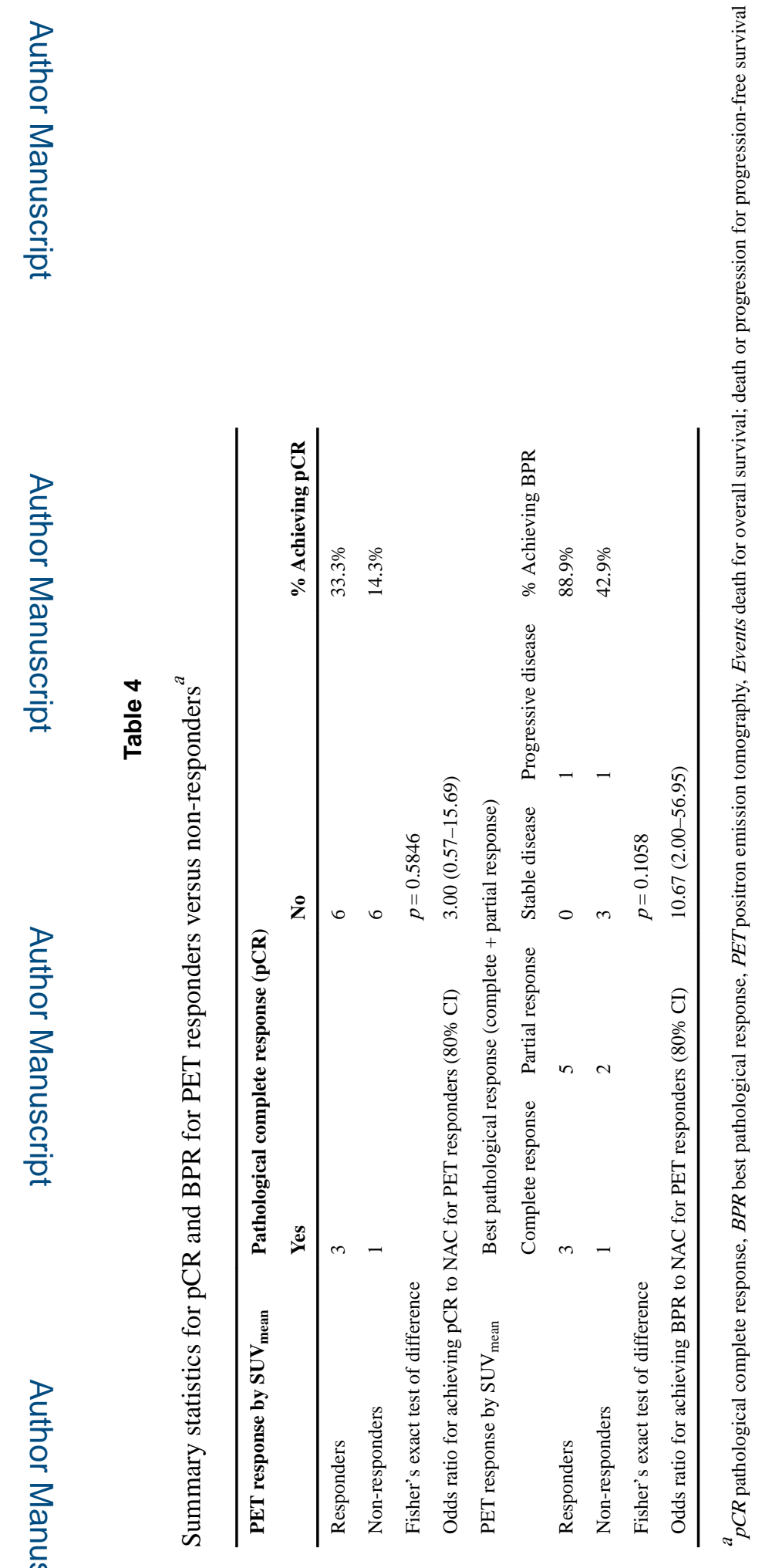

Ann Nucl Med. Author manuscript; available in PMC 2020 June 01. 